\title{
Focal Biologically Inspired Feature for Glaucoma Type Classification
}

\author{
Jun Cheng ${ }^{1}$, Dacheng Tao ${ }^{2}$, Jiang Liu ${ }^{1}$, Damon Wing Kee Wong ${ }^{1}$, \\ Beng Hai Lee ${ }^{1}$, Mani Baskaran ${ }^{3}$, Tien Yin Wong ${ }^{3,4}$, and Tin Aung ${ }^{3,4}$ \\ 1 Institute for Infocomm Research, Agency for Science, \\ Technology and Research, Singapore \\ \{jcheng, jliu, wkwong, benghai\}@i2r.a-star .edu.sg \\ 2 Centre for Quantum Computation and Intelligent Systems, FEIT, \\ University of Technology, Sydney, Australia \\ $\{$ dacheng.tao\}@uts.edu. au \\ 3 Singapore Eye Research Institute, Singapore \\ baskaran.mani@seri.com.sg, ophwty@nus.edu.sg, aung.tin@snec.com.sg \\ 4 Department of Ophthalmology, National University of Singapore, Singapore
}

\begin{abstract}
Glaucoma is an optic nerve disease resulting in loss of vision. There are two common types of glaucoma: open angle glaucoma and angle closure glaucoma. Glaucoma type classification is important in glaucoma diagnosis. Ophthalmologists examine the iridocorneal angle between iris and cornea to determine the glaucoma type. However, manual classification/grading of the iridocorneal angle images is subjective and time consuming. To save workload and facilitate large-scale clinical use, it is essential to determine glaucoma type automatically. In this paper, we propose to use focal biologically inspired feature for the classification. The iris surface is located to determine the focal region. The association between focal biologically inspired feature and angle grades is built. The experimental results show that the proposed method can correctly classify $85.2 \%$ images from open angle glaucoma and $84.3 \%$ images from angle closure glaucoma. The accuracy could be improved close to $90 \%$ with more images included in the training. The results show that the focal biologically inspired feature is effective for automatic glaucoma type classification. It can be used to reduce workload of ophthalmologists and diagnosis cost.
\end{abstract}

\section{Introduction}

Glaucoma is an optic nerve disease resulting in loss of vision. It is often associated with increased pressure of fluid inside the eye. Two common types of glaucoma are open angle glaucoma (OAG) and angle closure glaucoma (ACG). Ophthalmologists examine the iridocorneal angle between iris and cornea to determine OAG and ACG. When the angle is open, it is OAG. Otherwise, ACG. A detailed description of the angle structures can be found in 1 . Here we briefly explain why the iridocorneal angle is important. The iris, cornea, and lens are bathed in aqueous humor, which is continually produced by nearby tissues. It moves

G. Fichtinger, A. Martel, and T. Peters (Eds.): MICCAI 2011, Part III, LNCS 6893, pp. 91-98, 2011.

(C) Springer-Verlag Berlin Heidelberg 2011 
out of the eye via the trabecular meshwork drainage. Blocking of the trabecular meshwork would lead to increased pressure in the eye. The trabecular meshwork is associated with the angle, thus, the iridocorneal angle is important. Because of different causes and specific treatments for different types of glaucoma as well as the necessity of urgent treatment of ACG, it is important to determine the glaucoma type early [2, which implies that it is essential to visualize the iridocorneal angle to make a correct diagnosis of the disease.

Gonioscopy is an eye examination looking at the front part of the eye between iris and cornea. It requires considerable clinical expertise and effort as well as a full knowledge of the angle structures [3]. Thus, it is not performed as often as it should be. A new option with much more convenience is the RetCam (Clarity Medical Systems, Inc., Pleasanton, CA) camera, which is explored to capture the image of iridocorneal angle [3] recently. Four typical iridocorneal angle images from inferior, superior, nasal and temporal quadrants of a left eye captured by RetCam are shown in Fig. 1. The angle which is of our interest is located at the boundary between the iris and the cornea. When other angle structures are visible on the cornea side of the iris surface, it is an open angle, otherwise, closed. Shaffer's grading system [2] is widely used in gonioscopy to evaluate the angle status based on the visibility of the angle structures. In this paper, we focus on the clinically important grading: the classification between ACG and OAG. Manual grading of RetCam images is usually tedious, time consuming and expensive. Moreover, it is often subjective similar to many other medical applications 4] and thus reproducibility is a concern. To save workload and facilitate large-scale clinical use, it is essential to have a precise, efficient and cost effective system to determine glaucoma type automatically.

Automatic glaucoma type classification from iridocorneal images captured by RetCam is a new research topic and few work has been done for it. In [5], the

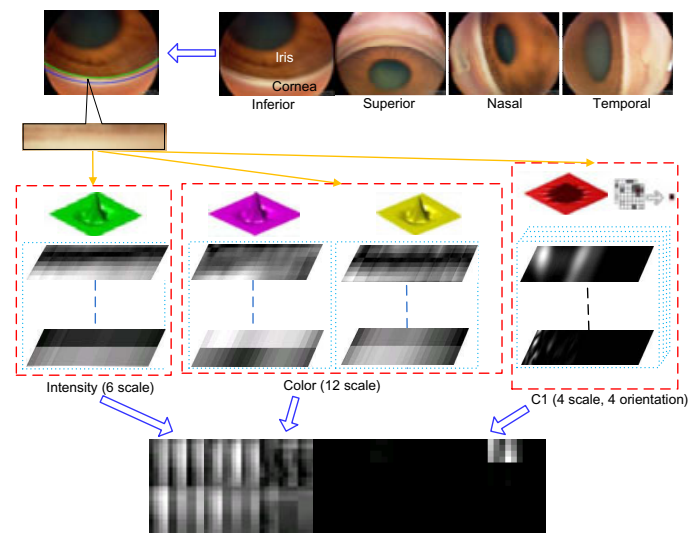

Fig. 1. Focal biologically inspired feature for glaucoma type classification: for each training image, we extract BIF from focal region. The feature consists of 6 intensity feature maps, 12 color feature maps, and $16 C 1$ units feature maps. 
edges around strongest arc are used. The limitation is that some edges within iris are mistaken as edges from angle structures and reduce the classification accuracy. Moreover, it relies on the accuracy of edge detection as well as the sensitivities used to determine the edges. Based on the observation that human can capture the 'gist' of an image instantly, biologically inspired feature (BIF) 6] 7] 8] has been used in computer vision including scene classification, gait recognition and etc. BIF mimics the process of cortex for visual perception. Since the ophthalmologists classify the two types of glaucoma based on the visual information, we introduce BIF to aid them to reduce workload. Different from the application of scene classification where the scene varies largely from one to another, the 'scene' of an angle closure image differs slightly from that of an open one in a small region. When ophthalmologists examine the images, they focus on the region. Thus, it is essential to extract BIF from it, i.e., focal BIF. The proposed focal BIF as shown in Fig. 1 simulates the process.

The paper is organized as follows. In Section 1 we have given an introduction of the background and motivation for the system. Section 2 introduces the BIF. In Section 3, we introduce the methodology to determine the focal region and extract focal BIF for angle grading. Section 4 shows the experimental results, followed by the conclusions in the last section.

\section{Biologically Inspired Feature}

The BIF has proven to be effective in computer vision. The feature consists of 34 feature maps including 6 feature maps from intensity units, 12 feature maps from color units, and 16 feature maps from $C 1$ units.

Among these features, the intensity units are obtained by convolving dyadic Gaussian pyramids with the intensity channel of a color image. The features correspond to the neurons of mammals which are sensitive to dark centers on bright surrounds or vice versa [9] 10]. Nine spatial scales are generated with a ratio from 1:1 (level 0) to 1:256 (level 8). The intensity feature maps are obtained by the center-surround difference operation between center levels $c=2,3,4$ and surround levels $s=c+d$, with $d=3,4$. Thus, six feature maps are computed at levels of 2-5, 2-6, 3-6, 3-7, 4-7, and 4-8. Because of the scale difference, maps of surround levels are interpolated to be the same size as the corresponding center levels, and then they are subtracted to generate the relevant feature maps, i.e., $I(c, s)=\mid I(c)-$ Interp $_{s-c} I(s) \mid$.

The color units are inspired by the 'color double-opponent' system in cortex 9]. Neurons are excited by a color (e.g., blue) and inhibited by another color (e.g., yellow) in the center of receptive field, so are neurons in the surround. Herein, four color channels are used: $R=r-(g+b) / 2, G=g-(r+b) / 2$, $B=b-(r+g) / 2$ and $Y=r+g-2(|r-g|+b)$. For each color channel $(R, G, B$, and $Y)$, dyadic Gaussian pyramids are used to generate nine spatial scales similar to intensity unit. Two color pairs $R-G$ and $B-Y$ are used. The feature maps are computed as the across scales center-surrounding differences. Similar to the computation of intensity units, surround maps are interpolated 
to be the same size as the corresponding center maps and their difference is computed as: $R G(c, s)=\mid(R(c)-G(c))-$ Interp $_{s-c}(R(s)-G(s)) \mid$ and $B Y(c, s)=$ $\mid(B(c)-Y(c))-$ Interp $_{s-c}(B(s)-Y(s)) \mid$.

The $C 1$ units are obtained by pooling over $S 1$ units, which correspond to simple cells in $S 1$ layer of the visual cortex. Gabor functions are used for feature extraction due to its similarity to the receptive field profiles in simple cells in $S 1$ layer. The Gabor mother function is given by: $F(x, y)=\exp \left(-\left(x_{0}^{2}+\right.\right.$ $\left.\left.\gamma^{2} y_{0}^{2}\right) /\left(2 \delta^{2}\right)\right) \times \cos \left(2 \pi x_{0} / \lambda\right)$, wherein $x_{0}=x \cos \theta+y \sin \theta, y_{0}=-x \sin \theta+y \cos \theta$, the range of $x$ and $y$ decides the scales of Gabor filters, and $\theta$ controls orientations. In this paper, eight scales with a range of sizes from $7 \times 7$ to $21 \times 21$ pixels with a step of two pixels are used. Four orientations are considered: $0^{\circ}$, $45^{\circ}, 90^{\circ}$, and $135^{\circ}$. Thus, a total of 32 feature maps are obtained in $S 1$ units. Pooling over two adjacent scales with an identical orientation, 16 feature maps are obtained from $C 1$ units.

Arbitrarily extracting the BIF from the whole image does not work well for angle grading as the main difference between ACG and OAG lies in a small region, not to mention the difference due to various quadrants. In order to use BIF properly, we propose focal BIF. Focal BIF refers to biologically inspired feature from a focal region. In this application, it is the area between iris and cornea.

\section{Methodology}

A system for automatic glaucoma type classification is proposed with following steps: quadrant determination, focal region segmentation, and grading.

\subsection{Quadrant Determination}

As mentioned earlier, the images can be from inferior, superior, nasal and temporal quadrant of the eye. One important step for the automated diagnosis is to determine the quadrant. In this paper, quadrant is determined based on the location of the arc center and the location of edges. Canny edge [11] followed by circular Hough transform [12] as in [5] are used to obtain the strongest arc. Assuming $\left(x_{i}, y_{i}\right), i=1,2, \cdots, N$ are the coordinates of all points from the arc inside the image, where top-left corner is defined as $(1,1)$ and bottom-right corner as $(m, n), N$ is the number of points. The function to determine the quadrant $Q$ is given as 1 :

$$
Q=\left\{\begin{array}{l}
\text { Superior, if } x_{c}-\bar{x}_{i} \geq\left|y_{c}-\bar{y}_{i}\right| \\
\text { Inferior, if } \bar{x}_{i}-x_{c} \geq\left|y_{c}-\bar{y}_{i}\right| \\
\text { Nasal, if } y_{c}-\bar{y}_{i}>\left|x_{c}-\bar{x}_{i}\right| \\
\text { Temporal, if } \bar{y}_{i}-y_{c}>\left|x_{c}-\bar{x}_{i}\right|
\end{array}\right.
$$

where $\left(\bar{x}_{i}, \bar{y}_{i}\right)=\left(\frac{1}{N} \sum_{i=1}^{N} x_{i}, \frac{1}{N} \sum_{i=1}^{N} y_{i}\right)$ is the mean of the edge coordinates, $\left(x_{c}, y_{c}\right)$ is the center of the detected arc.

\footnotetext{
${ }^{1}$ A left eye is assumed here, swop nasal and temporal for a right eye.
} 


\subsection{Focal Region Segmentation}

The focal region refers to the area human eye focused on when one examines the images. In this application, it is the area between iris and cornea. In order to extract focal region and align features from various regions, we propose to locate the iris surface as it is visible in both open angle and angle closure images. For angle closure, the iris surface is often the strongest ascending edge (from iris to cornea) in the focal region. However, for open angle, edges from other angle structures can be stronger. Thus, the output of the aforementioned circular Hough transform may find the iris surface inaccurately, as can be seen from the line in red in Fig. 2(a).

Without losing generality, assuming the image is from inferior side of an eye as in Fig. 2(a). Given $L_{j}(x)=I(x, j), x=1,2, \cdots, M$, from the $j^{\text {th }}$ column of the image $I$. Assuming $L_{j}$ crosses with the strongest arc at $x_{j}$. Inspired by the above observations on iris surface, we search for the point with strongest ascending edge (from iris to cornea) from pixels around $x_{j}$ in $L_{i}$ and get its coordinate $x_{k}$. Among all ascending Canny edge within $\left(x_{k}-w, x_{k}\right)$ as well as $x_{k}$ itself, the point closest to pupil is used as candidate iris surface point in this column. Here, $w$ is set to be estimated maximum angle width. Finally, curve fitting is applied based on all candidate points located in the last step. In this paper, the iris surface is modelled as part of circle and a circular Hough transform is applied again to find the fitted curve with circular center $\left(x_{c}, y_{c}\right)$ and radius $r$. After obtaining the estimation of iris surface highlighted in green, another circular arc can be determined based same circular center $\left(x_{c}, y_{c}\right)$ with a larger radius $r+\delta r$. The parameter $\delta r$ is set to be slightly larger than $w$. The region in between is the focal region. As the side portions are often blurred, central portion would be used. Locating the iris surface is a critical step to find the focal region and then the focal BIF. Visually, the above algorithm finds the iris surface accurately in central portion for 393 of the 396 images. However, it is difficult to get a ground truth to compute a quantitative accuracy.

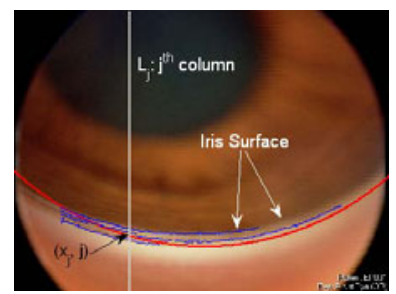

(a) Blue: Canny edge, red: detected arc

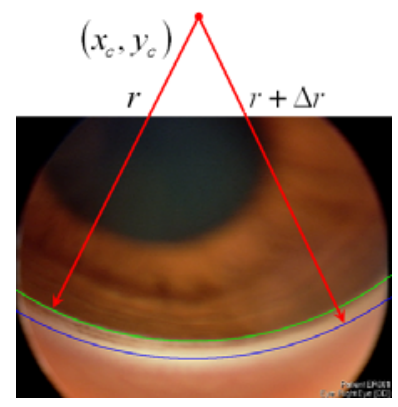

(b) Focal region

Fig. 2. Focal Region Segmentation 


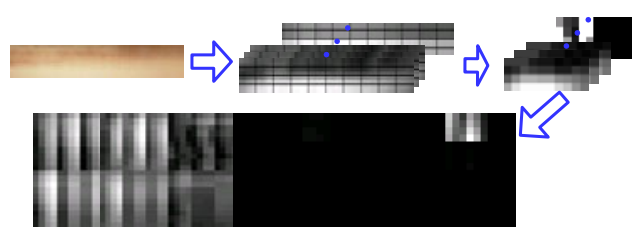

Fig. 3. Focal BIF from $4 \times 10$ sub-regions

A polar transform with respect to the circular center $\left(x_{c}, y_{c}\right)$ as in Fig. 2(b) is then applied to turn the focal region into rectangular. The transform helps to align the focal region from images at different quadrants. As ophthalmologists examine only the central portion, only the feature maps from central portion of the focal region are used. Each feature map is divided into $m \times n$ sub-regions. The mean value of feature map in each sub-region is computed for final representation. Fig. 3 shows an example with feature maps divided into $4 \times 10$ sub-regions. Since each image is represented by 34 feature maps and each feature map is decomposed into $m \times n$ sub-regions, we have a total of $34 m n$ mean values as the feature for each image.

\subsection{Grading}

In this paper, the grading problem is handled as a classification problem between ACG and OAG. Support vector machines (SVM) are used as the optimization tools for solving machine learning problems. The LIBSVM [13] is used in our experiments. RBF kernel is used with two-folder cross folder validation adopted to determine the parameters $C$ and $\gamma$.

\section{Experimental Results}

A total of 99 different patient eyes as in [5] are used. For each patient eye, four quadrants are examined by an ophthalmologist, thus a total of $99 \times 4=396$ quadrants are evaluated and labelled as ACG or OAG quadrant. Among the gradings, 166 quadrants are with ACG and 230 quadrants are with OAG. These manual gradings are used as ground truth.

In the SVM training, 115 images from OAG quadrants together with same number of images from ACG quadrants are randomly selected for the training. The rest of images are used for testing. The SVM parameters $C$ and $\gamma$ are determined automatically through cross validation [13. The training and testing are repeated five times to get an average result. Table 1 shows the percentage of corrected classified ACG and OAG by the proposed method for $m=4, n=10$ together the results by prior work [5] as well as the results when other classifiers are used. The results show conventional neural networks such as multi-layer perception (MLP), and k-nearest-neighbours ( $\mathrm{kNN}$ ) cannot perform as well as SVM on this task. To show the effectiveness of BIF, we also conduct tests by replacing the BIF with simplified features such as color histogram $(\mathrm{CH})$, color 
Table 1. Performance of the proposed method and other methods

\begin{tabular}{c|c|c|c|c}
\hline Method & Prior [5] & MLP & kNN & Proposed \\
\hline OAG & $80.3 \%$ & $77.4 \%$ & $51.8 \%$ & $85.2 \%$ \\
ACG & $80.3 \%$ & $74.3 \%$ & $65.0 \%$ & $84.3 \%$ \\
\hline
\end{tabular}

Table 2. Performance using other features

\begin{tabular}{c|c|c|c}
\hline Method & CH & CM & IH \\
\hline OAG & $59.0 \%$ & $65.1 \%$ & $54.2 \%$ \\
ACG & $67.5 \%$ & $57.8 \%$ & $56.6 \%$ \\
\hline
\end{tabular}

moment (CM) and intensity histogram (IH). The results summarized in Table 2 show that these features are not suitable for this task.

We also conduct tests with other $m$ and $n$ combinations. The classification accuracy is computed as the average accuracy of ACG and OAG and summarized in Fig. 4, The results show a slight performance drop with other settings. The average accuracy increases as $n$ increases to 10 . For larger $n$, it drops as too much redundant information leads to a biased classifier. A similar phenomena is observed as $m$ changes.

The two SVM parameters $C$ and $\gamma$ are important as well. Although $C$ and $\gamma$ are selected automatically, it is still necessary to look at the results with other parameters. Fig. 5 shows the performance with different $C$ and $\gamma$ combinations from exponentially growing $C$ and $\gamma$ for the case $m=4$ and $n=10$.

The number of training samples is another critical factor. Fig. 6] shows the results when different number of training images are used in the training. The results show that the accuracy can be improved close to $90 \%$ with more images included in the training.

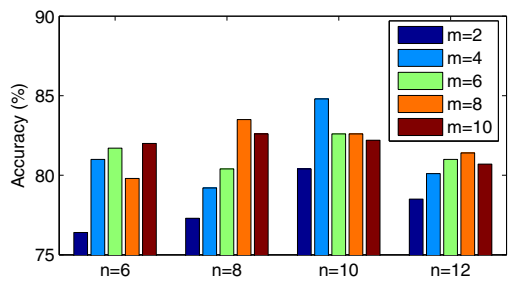

Fig. 4. Performance when each feature map is divided into $m \times n$ sub-regions

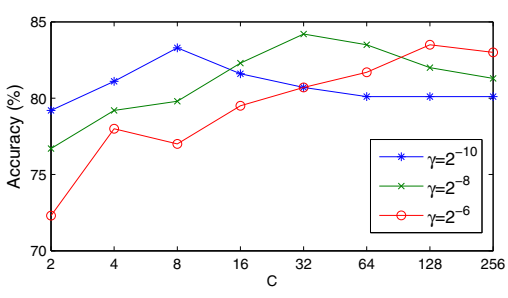

Fig. 5. Performance with various $C$ and $\gamma$

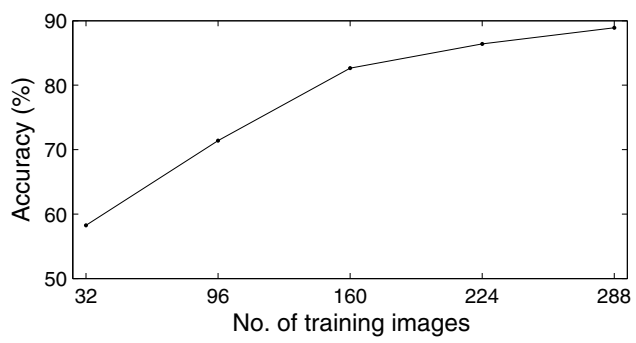

Fig. 6. Performance with different number of training images 


\section{Conclusion}

Glaucoma type classification is important in glaucoma diagnosis. Clinically, automatic glaucoma type classification helps reduce workload of ophthalmologists and diagnosis cost. It make it possible for large-scale clinical use and benefit patients. However, it is a challenging work due to ambiguous angle structures in some images. In this paper, we explore focal BIF for the classification. The association between focal BIF and glaucoma type is built through SVM learning. The results show that focal BIF is effective for the classification with $85.2 \%$ OAG and $84.3 \%$ ACG correctly detected based on $4 \times 10$ sub-regions. With more images included in the training, the accuracy can be improved close to $90 \%$. A limitation is that the ground truth used in this paper is from one ophthalmologist only and thus can be biased. In the future, gradings from more ophthalmologists are to be used. Future work would focus on feature representation to further improve the accuracy, with more images from different patients.

\section{References}

1. Minckler, D., Foster, P., Hung, P.T.: Angle Closure Glaucoma Classification and Racial Variation. Asia Journal of Ophthalmology 3(3,4), 3-9 (2001)

2. Salmon, J.F.: The Diagnostic Importance of Gonioscopy, http://www.glaucomaworld.net/english/019/e019a01t.html/

3. Ahmed, I.I.K., Mackeen, L.D.: A New Approach for Imaging the Angle. Glaucoma Today, 27-30 (2007)

4. Huang, W., Li, H., Chan, K.L., Lim, J.H., Liu, J., Wong, T.Y.: A Computer Aided Diagnosis System of Nuclear Cataract via Ranking. In: Yang, G.-Z., Hawkes, D., Rueckert, D., Noble, A., Taylor, C. (eds.) MICCAI 2009. LNCS, vol. 5762, pp. 803-810. Springer, Heidelberg (2009)

5. Cheng, J., Liu, J., Lee, B. H., Wong, D., Yin, F., Aung, T., Baskaran, M., Perera, S., Wong, T.Y.: Closed Angle Glaucoma Detection in Retcam Images. In: Proc. of Int. Conf. of the IEEE Engineering in Med. and Bio. Society, pp. 4096-4099 (2010)

6. Siagian, C., Itti, L.: Rapid Biologically-inspired Scene Classification using Features Shared with Visual Attention. IEEE Trans. Pattern Anal. Mach. Intell. 31(2), 260 274 (2008)

7. Song, D., Tao, D.: Biologically Inspired Feature Manifold for Scene Classification. IEEE Trans. Image Processing 19(1), 174-184 (2010)

8. Mu, Y., Tao, D.: Biologically Inspired Feature Manifold for Gait Recongition. Neurocomputing 73, 895-902 (2010)

9. Itti, L., Koch, C., Niebur, E.: A Model of Saliency-based Visual Attention for Rapid Scene Analysis. IEEE Trans. Pattern Anal. Mach. Intell. 20(11), 1254-1259 (1998)

10. Leventhal, A.G.: The Neural Nasis of Visual Function. Vision and Visual Dysfunction (1991)

11. Canny, J.: A Computational Approach to Edge Detection. IEEE Trans. Pattern Anal. and Mach. Intell. 8, 679-698 (1986)

12. Morse, B.S.: Segmentation (Edge based, Hough Transform). Brigham Young University, Lecture Notes (2000)

13. Chang, C.C., Lin, C.J.: LIBSVM: A Library for Support Vector Machines, http://www.csie.ntu.edu.tw/ cjlin/libsvm 